\title{
Reservoritis, una complicación del tratamiento quirúrgico de la colitis ulcerativa. Diagnóstico y tratamiento. Revisión
}

\section{Pouchitis, a complication associated with the surgical treatment of ulcerative colitis. Diagnosis and treatment. Review}

William Otero-Regino, ${ }^{1 *}$ (]) Lina Otero-Parra, ${ }^{2}$ Guillermo Veitía. ${ }^{3}$

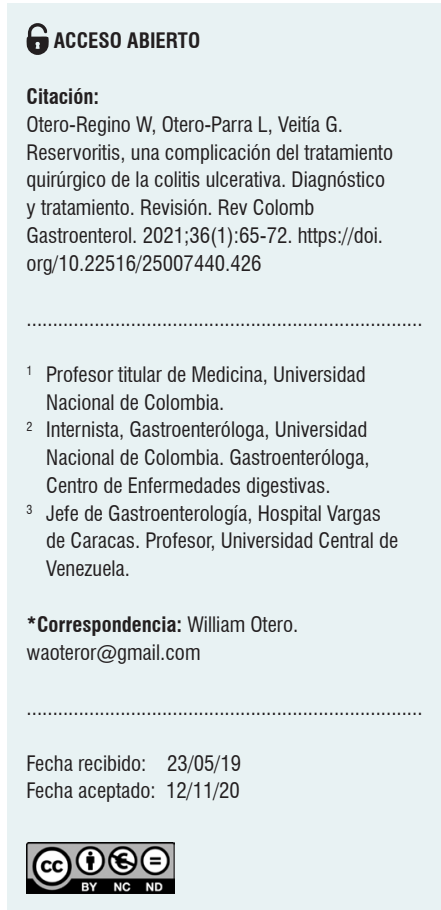

\begin{abstract}
Resumen
Del $20 \%$ al $30 \%$ de los pacientes con colitis ulcerativa (CU) son sometidos a cirugía, ya sea por intratabilidad, curso fulminante, aparición de displasia o cáncer de colon. La cirugía de elección es la proctocolectomía con reservorio ileoanal. Sin embargo, el $20 \%-50 \%$ de los pacientes presentan reservoritis a 10 años. El diagnóstico se realiza con base en las manifestaciones clínicas, las alteraciones endoscópicas y la histología. Los medicamentos utilizados en el tratamiento de la CU no son tan eficaces en la reservoritis y el tratamiento de primera línea es los antibióticos. Dependiendo de la respuesta inicial y el curso clínico de la entidad, se elegirá el tratamiento posterior. En esta revisión se discuten los aspectos más importantes con respecto a la epidemiología, diagnóstico, patogénesis y tratamiento de la reservoritis.
\end{abstract}

\section{Palabras claves}

Colitis ulcerativa, reservoritis, antibióticos, profilaxis.

\begin{abstract}
Between $20 \%$ and $30 \%$ of patients with ulcerative colitis (UC) undergo surgery because it is not treatable or because it is associated with a fulminant course, dysplasia, or colon cancer. The surgery of choice is proctocolectomy with ileal-anal pouch. However, $20 \%-50 \%$ of patients present with pouchitis 10 years after surgery. The diagnosis is made based on clinical manifestations, endoscopic alterations, and histology. The drugs used in the treatment of UC are not as effective in pouchitis and the first-line treatment is antibiotics administration. Depending on the initial response and clinical course of the condition, subsequent treatment will be chosen. This review discusses the most important aspects of the epidemiology, diagnosis, pathogenesis, and treatment of pouchitis.
\end{abstract}

Keywords

Ulcerative colitis, Pouchitis, Antibiotics, Prophylaxis.

\section{INTRODUCCIÓN}

La colitis ulcerativa (CU) es una entidad autoinflamatoria, multifactorial, que produce inflamación crónica de la mucosa del colon, con frecuentes manifestaciones extraintestinales y un curso clínico variable con frecuentes recaídas (1). Con el enfoque terapéutico actual, la necesidad de cirugía ha disminuido, pero todavía el $20 \%$ (2) al $30 \%$ (3) de los pacientes deben ser operados, ya sea por intratabilidad, curso fulminante, aparición de displasia o cáncer de colon $(2,3)$. La cirugía de elección es la proctocolectomía con reservorio ileoanal (4), descrita por primera vez por Park y colaboradores en 1978 (5). En el $93 \%$ de los pacientes, el reservorio es funcionante después de 30 años de la cirugía (6). Los principales tipos de reservorio son en "J" en "W" y en "S" (Figura 1) (7). El más frecuentemente realizado es el que tiene forma de "J" (7). 


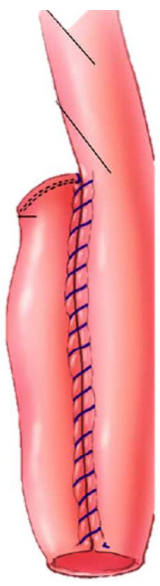

En "J"

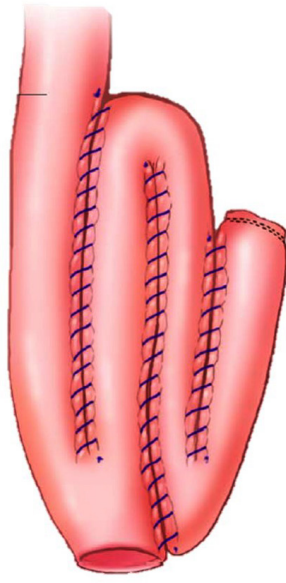

En "W"

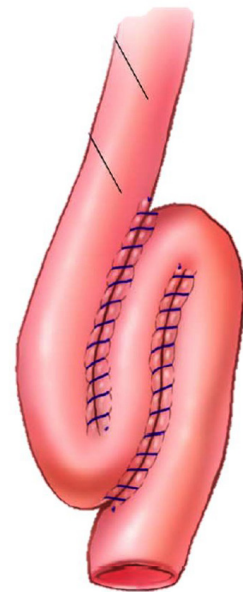

En "S"
Figura 1. Principales tipos de reservorio. Modificado de referencia 7.

Después de la cirugía, el reservorio puede presentar inflamación persistente conocida como reservoritis, así como también complicaciones no inflamatorias (Tabla 1) (4-6). En este artículo se revisa específicamente la reservoritis, por ser la complicación más frecuente relacionada con el reservorio ileoanal $(5,6)$.

Tabla 1. Diagnóstico diferencial de reservoritis

Inflamación de la mucosa rectal remanente (cuffitis)
Diarrea infecciosa por CMV o C. difficile
Estenosis del reservorio
Isquemia del reservorio
Enfermedad de Crohn del reservorio (habrá alteraciones inflamatorias
en áreas proximales)
Adherencias
Disfunción del piso pélvico
Mala absorción de sales biliares
Alteración del vaciamiento del reservorio
Reservoritis autoinmune
EPIDEMIOLOGÍA

La incidencia de reservoritis varía en el tiempo. $\mathrm{Al}$ año, a 5 y a 10 años, la incidencia es de $20 \%, 40 \%$ y $50 \%$, respectivamente (8). A los 30 años de la cirugía, puede afectar al $80 \%$ de los pacientes (6). Esas cifras contrastan con la baja incidencia de reservoritis (0\%-10\%) en los pacientes operados con la misma cirugía por poliposis adenomatosa familiar (9).

\section{PATOGÉNESIS}

No se conocen con exactitud los mecanismos fisiopatológicos que originan la reservoritis. Sin embargo, es más probable que sea una entidad multifactorial que involucra predisposición genética, sobrecrecimiento bacteriano y disbiosis en el reservorio ileal, entre otros (9). Recientemente, se ha identificado que en la reservoritis aguda hay un aumento de especies del género Clostridia y, recíprocamente, su disminución se asocia con la respuesta al tratamiento antibiótico (10). En cambio, en la reservoritis crónica hay un aumento de Staphylococcus aureus (10). Otros factores implicados pueden ser la disminución de ácidos grasos de cadena corta, deficiencias nutricionales, isquemia o alteración de la respuesta inmune (10-13). Adicionalmente, otros autores han considerado que la reservoritis puede ser una enfermedad inflamatoria intestinal diferente (14) y diversos factores aumentan el riego de esta entidad (15-18). Los antiinflamatorios no esteroideos (AINE) aumentan el riesgo 3,24 veces (intervalo de confianza [IC] $95 \%: 1,71-6,13)(15)$.

Similar a la colitis ulcerativa, se ha encontrado que el tabaquismo tiene una asociación negativa y dejar de fumar aumenta el riesgo, pero esto no significa que las personas deberían fumar para evitar la enfermedad, ya que el tabaquismo tiene múltiples efectos nocivos en el ser humano $y$, recientemente, se ha encontrado que el tabaquismo activo no evita esta complicación (16). Cuando hay colangitis esclerosante primaria concurrente con la $\mathrm{CU}$, el riesgo se aumenta con respecto a la CU sin esa complicación (17). Los altos niveles de anticuerpo anticitoplasma de neutrófilo de tinción perinuclear ( $\mathrm{p}$-ANCA) confieren un riesgo de reservoritis de 8,5 (17). Un reciente metaanálisis encontró que la presencia de p-ANCA se asociaba con mayor riesgo de reservoritis crónica (Odds ratio [OR]: 1,8; IC 95 \%: 1,2$2,6)$, pero no para reservoritis aguda (18). Una crítica a ese metaanálisis es que, en la mayoría de los estudios incluidos, la determinación de p-ANCA se realizó después de la cirugía.

\section{DIAGNÓSTICO}

El diagnóstico se realiza con base en la combinación de manifestaciones clínicas, alteraciones endoscópicas (reservorioscopia) y hallazgos histológicos (19, 20). Para el diagnóstico se necesitan mínimo 7 puntos (21). La gravedad puede ser leve, moderada o grave (19). En algunos casos se necesitan estudios de imágenes de abdomen y pelvis $(20$, 22). No existen manifestaciones clínicas específicas de la reservoritis (12). Clínicamente, hay aumento de las deposiciones, acompañadas de moco, dolor abdominal, tenesmo, incontinencia y síntomas nocturnos $(12,22)$. El sangrado rectal es más sugestivo de inflamación de la mucosa rectal remanente (cuffitis) (20). La inflamación de esa mucosa 
rectal puede ser en realidad una recurrencia de la colitis ulcerativa (20). Las manifestaciones sistémicas como fiebre, escalofríos y pérdida de peso sugieren más frecuentemente una infección del reservorio (reservoritis infecciosa) por Clostridium difficile o citomegalovirus (CMV) $(12,20)$. Usualmente, los pacientes con reservorio ileoanal tienen 4 a 7 movimientos intestinales al día $(12,22)$ e incluso hasta 20, por lo cual el aumento de defecaciones por sí solo no indica reservoritis. El diagnóstico diferencial incluye diversas entidades (Tabla 1) $(12,20,22)$.

En la endoscopia se encuentra eritema, edema, friabilidad, granularidad, sangrado espontáneo o al contacto con el endoscopio, úlceras, pólipos inflamatorios, puentes mucosos y disminución de la distensibilidad (22). La presencia de úlceras o erosiones en la anastomosis no indica reservoritis (20). Las biopsias se deben tomar del reservorio (pouch) y del asa proximal del íleon, pero no de la línea de sutura (20). No obstante que la inflamación está en el reservorio ileal, ocasionalmente la inflamación se puede extender más proximalmente y afectar el íleon proximal al reservorio $(12,20)$. El compromiso del íleon proximal al reservorio se conoce como ileitis preservorio, fue descrita por primera vez por Bell y colaboradores (23) en un estudio retrospectivo de pacientes con CU que habían sido tratados quirúrgicamente y se les había practicado colectomía total con reservorio ileoanal. En la mitad de sus pacientes había concomitantemente reservoritis y en un caso la histopatología mostró granulomas, lo cual es muy importante ya que erróneamente se puede diagnosticar enfermedad de Crohn (23).

En las biopsias se encuentra infiltrado inflamatorio con polimorfonucleares, abscesos de las criptas y úlceras (23, 24). Adicionalmente, se encuentran cambios crónicos, aplanamiento de las vellosidades de la mucosa ileal que lleva a atrofia vellosa (metaplasia colónica) e infiltrado inflamatorio crónico $(12,20,22)$. Si no hay cambios inflamatorios agudos, no se puede diagnosticar reservoritis, ya que la estasis crónica de materia fecal, en el reservorio, produce cambios inflamatorios crónicos $(25,26)$. Hallazgos sugestivos de infección por CMV son las inclusiones basófilas y eosinófilas $(12,20)$. El diagnóstico final de esta infección se basa en la demostración del CMV por inmunohistoquímica $(20,22)$. La apoptosis en las criptas sugiere reservoritis autoinmune (27). La inflamación no homogénea o asimétrica sugiere reservoritis isquémica $(12,20,27)$. Cuando se identifica estenosis del reservorio, la dilatación endoscópica con balón es el tratamiento de elección por su eficacia y seguridad (28).

\section{RIESGO DE DISPLASIA Y CÁNCER}

El riesgo de displasia en el reservorio es extremadamente raro cuando no hay neoplasia o displasia preoperatoria (29).
En un estudio se encontró que 10 a 15 años poscirugía esa alteración se produjo en menos del $1 \%$ de los pacientes (29) y en una investigación más reciente, el riesgo de cáncer fue de $1,3 \%$ (30). En cambio, cuando existe displasia o cáncer antes de la cirugía, el riesgo de neoplasia aumenta sustancialmente de la siguiente manera: con displasia, el Hazard ratio (HR) es de 3,76 (IC $95 \%: 1,39-10,19)$ y con cáncer previo, el HR es de 24,69 (IC $95 \%$ : 9,61-63,42) (30). En estos pacientes, la recomendación es vigilar anualmente el reservorio con endoscopia. En pacientes con antecedente de CU de 10 años de evolución antes del reservorio y uno de los siguientes factores: reservoritis crónica, cuffitis, historia familiar de cáncer de colon en familiares de primer grado o colangitis esclerosante primaria, se recomienda endoscopia de vigilancia del reservorio con biopsias cada 1-3 años (20). Cuando no existen los factores de riesgo mencionados, la vigilancia se hace cada 3 años, comenzando 10 años después del diagnóstico de la CU (20).

\section{CLASIFICACIÓN Y CURSO CLÍNICO}

En la Tabla 2 se muestra la clasificación de la reservoritis con base en el examen endoscópico, histología, curso clínico, duración, respuesta a antibióticos y número de episodios al año $(22,31,32)$.

Tabla 2. Tipos de reservoritis

\begin{tabular}{ll}
\hline Evolución & $\begin{array}{l}\text { Aguda: }<4 \text { semanas } \\
\text { Crónica: }>4 \text { semanas, } 10 \%-15 \%\end{array}$ \\
\hline $\begin{array}{l}\text { Respuesta a } \\
\text { antibióticos }\end{array}$ & $\begin{array}{l}\text { Respondedor a antibióticos } \\
\text { No respondedor a antibióticos } \\
\text { Dependiente de antibióticos (3 episodios al año) } \\
\text { Refractaria a antibióticos }\end{array}$ \\
\hline $\begin{array}{l}\text { Episodios al } \\
\text { año }\end{array}$ & $\begin{array}{l}\text { Infrecuente: } 3 \text { episodios al año } \\
\text { Frecuente: }>3 \text { episodios al año }\end{array}$ \\
\hline $\begin{array}{l}\text { Secundaria } \\
\text { a otra } \\
\text { patología }\end{array}$ & $\begin{array}{l}\text { Isquemia, CMV, autoinmune asociada con lgG4, } \\
\text { deficiencia de ácidos grasos de cadena corta, } \\
\text { enfermedad de Crohn, C. difficile, AINE. Estas } \\
\text { categorías no son mutuamente excluyentes. }\end{array}$ \\
\hline
\end{tabular}

IgG4: inmunoglobulina G 4.

\section{TRATAMIENTO}

Los medicamentos clásicamente utilizados en el tratamiento de la colitis ulcerativa no tienen eficacia similar en reservoritis $(7,22,33,34)$. La piedra angular del manejo son los antibióticos y la decisión de estrategias adicionales, depende del fracaso de esta terapia de primera línea $(7,12-14,20,22)$. Teniendo en cuenta su alta incidencia y su curso clínico, el manejo puede dividirse en profilaxis 
primaria con probióticos y tratamiento de la reservoritis aguda con antibióticos $(7,12-14,20)$. De acuerdo con la respuesta a los antibióticos, los pacientes se clasifican como respondedores a antibióticos, dependientes de antibióticos (3 episodios al año) y refractarios a los antibióticos (12-14, $20,22)$. Este último grupo se trata con una combinación de antibióticos de manera escalonada: budesonida, terapia biológica con antifactor de necrosis tumoral (anti-TNF: infliximab, adalimumab) o antiintegrinas (vedolizumab), e incluso altas dosis de probióticos a largo plazo. El $60 \%-80 \%$ de los pacientes refractarios obtiene beneficios con esta estrategia escalonada (step up). Sin embrago, $1 \%-2 \%$ de los pacientes termina con ileostomía permanente (22).

El manejo de la entidad se puede dividir en profilaxis primaria, tratamiento de la reservoritis aguda, tratamiento de la reservoritis dependiente de antibióticos y tratamiento de la reservoritis crónica.

\section{Profilaxis primaria}

Diversos estudios han investigado probióticos para prevenir la aparición de reservoritis. Sin embargo, los resultados son inconsistentes $y$, por tanto, no hay evidencia definitiva sobre su eficacia $(31,35-39)$. En dos estudios se encontró una menor incidencia de reservoritis en el grupo que recibió probióticos $(34,35,40)$. En el estudio de Gionchetti (35), con 40 pacientes, la reservoritis ocurrió en el $10 \%$ de los que recibieron probióticos, frente al $40 \%$ del grupo con placebo. En el estudio de Yasueda (40), con 17 pacientes, al final de dos años de seguimiento, 1 paciente de 9 del grupo probiótico presentó reservoritis, frente a 4 de 8 del grupo placebo. Por el contrario, en otro estudio, con 30 pacientes, no hubo diferencia significativa en los dos grupos (40).

\section{Tratamiento de reservoritis aguda}

La piedra angular es los antibióticos (7). El antibiótico recomendado inicial es ciprofloxacina $500 \mathrm{mg}$ cada 12 horas por 14 días $(12,14,20,22)$. Otras opciones pueden ser el metronidazol o tinidazol, $500 \mathrm{mg}$ cada 12 horas $(12,14,22$, 40). La mayoría de los pacientes responden rápidamente a este esquema. Se prefiere la ciprofloxacina por su mayor eficacia y porque tiene menos efectos colaterales $(12,22)$. La mayoría de los pacientes responden a este tratamiento empírico inicial; sin embargo, el $60 \%$ tiene por lo menos una recurrencia (22). La ciprofloxacina es más eficaz que el metronidazol (100 \% frente al $33 \%$ ) (22). La respuesta a los antibióticos respalda el concepto de que la disbiosis puede estar involucrada. Los pacientes que no responden al esquema inicial con antibióticos pueden recibir un nuevo ciclo de los mismos durante 4 semanas, ya sea con base en los resultados del cultivo, o empíricamente combinando ciprofloxacina $500 \mathrm{mg}$ cada 12 horas con metronidazol o tinidazol, cada uno $500 \mathrm{mg}$ cada 12 horas o con rifaximina $550 \mathrm{mg}$ cada 12 horas $(12,20,22,40)$. Con base en la respuesta o no a los antibióticos, los pacientes pueden clasificarse prospectivamente, como se describe a continuación.

\section{Reservoritis dependiente de antibióticos}

Este cuadro corresponde a los pacientes que tienen una respuesta inicial a los antibióticos, pero tienen 3 o más recaídas al año. La recomendación para ellos es dar antibióticos permanentes a dosis bajas, como ciprofloxacina 200 a $500 \mathrm{mg}$ /día o rifaximina 200 a $550 \mathrm{mg}$ una o dos veces al día. En un reciente estudio, solo el $21 \%$ de los pacientes que recibieron antibióticos crónicamente tuvo mejoría al año de tratamiento, el $28 \%$ tuvo efectos adversos por los antibióticos y en el $78 \%$ de los pacientes se identificó resistencia antimicrobiana (33). Esta baja eficacia plantea la necesidad de tratamientos diferentes, incluidos los biológicos e incluso probióticos con VSL \# 3 en dosis de 6-9 g/ día $(12,20,22)$.

\section{Reservoritis crónica refractaria a antibióticos}

No existe una definición unánime. Sin embargo, se considera que son los pacientes que tienen síntomas por lo menos 4 semanas y no respondieron al manejo antibiótico. De $10 \%$ $15 \%$ de los pacientes presentan este cuadro. En esos casos, es necesario descartar las causas secundarias no inflamatorias que incluyen las mecánicas (isquemia, estenosis, fístulas, falta de la punta de la "J", entre otras) (22). Siempre es necesario descontinuar los AINE. En casos de infección por CMV, el tratamiento sería ganciclovir $(22,31)$. Si no hay causas secundarias, el manejo podría ser con inmunosupresores que incluyen budesonida o betametasona $(12,22)$; y otra opción para estos pacientes al igual que para los que son dependientes de antibióticos sería utilizar terapias biológicas como anti-TNF (infliximab o adalimumab) (41) o vedolizumab (42). En reportes de casos, se ha encontrado beneficio con ustekinumab (43) y en casos refractarios recientemente se ha encontrado beneficio con tofacitinib (44).

Cuando hay inflamación en el reservorio y también en el íleon proximal, el manejo de elección es budesonida oral $(12,22)$. La cuffitis o inflamación de la mucosa rectal remanente da un cuadro similar a la reservoritis, excepto porque presenta sangrado más frecuentemente $(12,20)$. El manejo de elección es con ácido 5-aminosalicílico (5-ASA) tópico (supositorios) y, si no hay respuesta, se recomienda combinar el tratamiento con mesalazina oral $(20,22)$. Un algoritmo sobre el manejo general se muestra en la Figura 2. 


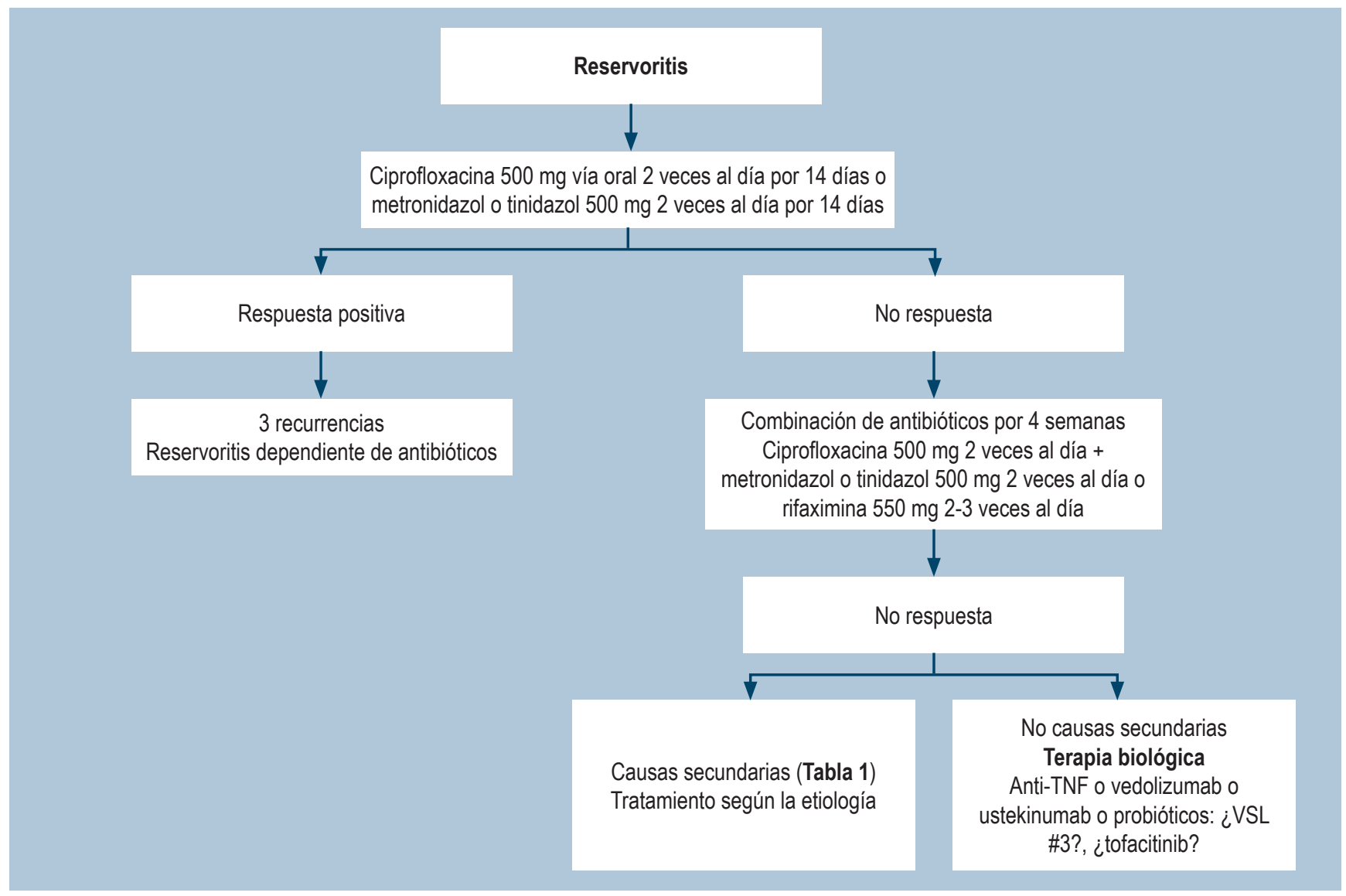

Figura 2. Manejo de la reservoritis.

El tratamiento quirúrgico es una opción de rescate cuando fracasa el tratamiento médico (34). La cirugía tiene el propósito de resecar el reservorio (reservoriectomía) e ileostomía definitiva $(7,22,34)$. Se desconoce la proporción de pacientes en los cuales esta cirugía es realizada por pérdida del reservorio, pero se estima que puede ser necesaria en el $25 \%$ de los pacientes $(45,46)$.
La cuffitis refractaria a tratamiento médico con frecuencia se puede acompañar de isquemia y tanto estas como la cuffitis colágena se consideran candidatos a mucosectomía transanal o incluso desfuncionalización del reservorio $(7,22,34)$.

El trasplante de materia fecal se ha utilizado en casos de reservoritis crónica refractaria; sin embrago, hasta el momento la evidencia disponible es escasa y limitada a series de casos (47).

\section{REFERENCIAS}

1. Park H, Bourla AB, Kastner DL, Colbert RA, Siegel RM. Lighting the fires within: the cell biology of autoinflammatory diseases. Nat Rev Immunol. 2012;12(8):570-80. https://doi.org/10.1038/nri3261

2. Parragi L, Fournier N, Zeitz J, Scharl M, Greuter T, Schreiner P, Misselwitz B, Safroneeva E, Schoepfer AM, Vavricka SR, Rogler G, Biedermann L; Swiss IBD
Cohort Study Group. Colectomy Rates in Ulcerative Colitis are Low and Decreasing: 10-year Follow-up Data From the Swiss IBD Cohort Study. J Crohns Colitis. 2018;12(7):811-818. https://doi.org/10.1093/ecco-jcc/ jjy040

3. Frolkis AD, Dykeman J, Negrón ME, Debruyn J, Jette N, Fiest KM, Frolkis T, Barkema HW, Rioux KP, Panaccione 
R, Ghosh S, Wiebe S, Kaplan GG. Risk of surgery for inflammatory bowel diseases has decreased over time: a systematic review and meta-analysis of population-based studies. Gastroenterology. 2013;145(5):996-1006. https:// doi.org/10.1053/j.gastro.2013.07.041

4. Parks AG, Nicholls RJ. Proctocolectomy without ileostomy for ulcerative colitis. Br Med J. 1978;2(6130):85-8. https://doi.org/10.1136/bmj.2.6130.85

5. Fazio VW, Kiran RP, Remzi FH, Coffey JC, Heneghan HM, Kirat HT, Manilich E, Shen B, Martin ST. Ileal pouch anal anastomosis: analysis of outcome and quality of life in 3707 patients. Ann Surg. 2013;257(4):679-85. https://doi. org/10.1097/SLA.0b013e31827d99a2

6. Lightner AL, Mathis KL, Dozois EJ, Hahnsloser D, Loftus EV Jr, Raffals LE, Pemberton JH. Results at Up to 30 Years After Ileal Pouch-Anal Anastomosis for Chronic Ulcerative Colitis. Inflamm Bowel Dis. 2017;23(5):781-790. https:// doi.org/10.1097/MIB.0000000000001061

7. Quinn KP, Raffals LE. An Update on the Medical Management of Inflammatory Pouch Complications. Am J Gastroenterol. 2020;115(9):1439-1450. https://doi. org/10.14309/ajg.0000000000000666

8. Athayde J, Davies SC, Parker CE, Guizzetti L, Ma C, Khanna R, Feagan BG, Jairath V. Placebo Rates in Randomized Controlled Trials of Pouchitis Therapy. Dig Dis Sci. 2018;63(10):2519-2528. https://doi. org/10.1007/s10620-018-5199-9

9. Schieffer KM, Williams ED, Yochum GS, Koltun WA. Review article: the pathogenesis of pouchitis. Aliment Pharmacol Ther. 2016;44(8):817-35. https://doi. org/10.1111/apt.13780

10. Segal JP, Oke S, Hold GL, Clark SK, Faiz OD, Hart AL. Systematic review: ileoanal pouch microbiota in health and disease. Aliment Pharmacol Ther. 2018;47(4):466-477. https://doi.org/10.1111/apt.14454

11. Seril DN, Yao Q Shen B. The association between autoimmunity and pouchitis. Inflamm Bowel Dis. 2014;20(2):378-88. https://doi.org/10.1097/01. MIB.0000435761.82705.6a

12. Shen B. Pouchitis: what every gastroenterologist needs to know. Clin Gastroenterol Hepatol. 2013;11(12):1538-49. https://doi.org/10.1016/j.cgh.2013.03.033

13. Rothenberger DA. Pouchitis and empiricism: can we progress? Mayo Clin Proc. 1994;69(5):491-2. https://doi. org/10.1016/s0025-6196(12)61649-8

14. Cheifetz A, Itzkowitz S. The diagnosis and treatment of pouchitis in inflammatory bowel disease. J Clin Gastroenterol. 2004;38(5 Suppl 1):S44-50. https://doi. org/10.1097/01.mcg.0000124001.93146.ef

15. Shen B, Fazio VW, Remzi FH, Brzezinski A, Bennett AE, Lopez R, Hammel JP, Achkar JP, Bevins CL, Lavery IC, Strong SA, Delaney CP, Liu W, Bambrick ML, Sherman KK, Lashner BA. Risk factors for diseases of ileal pouchanal anastomosis after restorative proctocolectomy for ulcerative colitis. Clin Gastroenterol Hepatol. 2006;4(1):81-9; quiz 2-3. https://doi.org/10.1016/j.cgh.2005.10.004
16. Gorrepati VS, Stuart A, Deiling S, Koltun W, Tinsley A, Williams ED, Coates MD. Smoking and the Risk of Pouchitis in Ulcerative Colitis Patients With Ileal PouchAnal Anastomosis. Inflamm Bowel Dis. 2018;24(9):20272032. https://doi.org/10.1093/ibd/izy097

17. Fleshner PR, Vasiliauskas EA, Kam LY, Fleshner NE, Gaiennie J, Abreu-Martin MT, Targan SR. High level perinuclear antineutrophil cytoplasmic antibody (pANCA) in ulcerative colitis patients before colectomy predicts the development of chronic pouchitis after ileal pouchanal anastomosis. Gut. 2001;49(5):671-7. https://doi. org/10.1136/gut.49.5.671

18. Singh S, Sharma PK, Loftus EV Jr, Pardi DS. Meta-analysis: serological markers and the risk of acute and chronic pouchitis. Aliment Pharmacol Ther. 2013;37(9):867-75. https://doi.org/10.1111/apt.12274

19. Shen B, Achkar JP, Lashner BA, Ormsby AH, Remzi FH, Bevins CL, Brzezinski A, Petras RE, Fazio VW. Endoscopic and histologic evaluation together with symptom assessment are required to diagnose pouchitis. Gastroenterology. 2001;121(2):261-7. https://doi. org/10.1053/gast.2001.26290

20. Barreiro-de Acosta M, Gutierrez A, Rodríguez-Lago I, Espín E, Ferrer Bradley I, Marín-Jimenez I, Beltrán B, Chaparro M, Gisbert JP, Nos P; en representación de GETECCU. Recommendations of the Spanish Working Group on Crohn's Disease and Ulcerative Colitis (GETECCU) on pouchitis in ulcerative colitis. Part 1: Epidemiology, diagnosis and prognosis. Gastroenterol Hepatol. 2019;42(9):568-578. https://doi.org/10.1016/j. gastrohep.2019.08.001

21. Sandborn WJ, Tremaine WJ, Batts KP, Pemberton JH, Phillips SF. Pouchitis after ileal pouch-anal anastomosis: a Pouchitis Disease Activity Index. Mayo Clin Proc. 1994;69(5):409-15. https://doi.org/10.1016/s00256196(12)61634-6

22. Shen BO. Management of acute and chronic pouchitis [internet]. Up To date; 2020 [acceso el 25 de julio de 2020]. Disponible en: https://www.uptodate.com/contents/management-of-acute-and-chronic-pouchitis

23. Bell AJ, Price AB, Forbes A, Ciclitira PJ, Groves C, Nicholls RJ. Pre-pouch ileitis: a disease of the ileum in ulcerative colitis after restorative proctocolectomy. Colorectal Dis. 2006;8(5):402-10. https://doi.org/10.1111/j.14631318.2006.00954.x

24. Shepherd NA, Jass JR, Duval I, Moskowitz RL, Nicholls RJ, Morson BC. Restorative proctocolectomy with ileal reservoir: pathological and histochemical study of mucosal biopsy specimens. J Clin Pathol. 1987;40(6):601-7. https://doi.org/10.1136/jcp.40.6.601

25. de Silva HJ, Millard PR, Kettlewell M, Mortensen NJ, Prince C, Jewell DP. Mucosal characteristics of pelvic ileal pouches. Gut. 1991;32(1):61-5. https://doi.org/10.1136/ gut.32.1.61

26. Shepherd NA, Healey CJ, Warren BF, Richman PI, Thomson WH, Wilkinson SP. Distribution of mucosal 
pathology and an assessment of colonic phenotypic change in the pelvic ileal reservoir. Gut. 1993;34(1):101-5. https://doi.org/10.1136/gut.34.1.101

27. Jiang W, Goldblum JR, Lopez R, Lian L, Shen B. Increased crypt apoptosis is a feature of autoimmune-associated chronic antibiotic refractory pouchitis. Dis Colon Rectum. 2012;55(5):549-57. https://doi.org/10.1097/ DCR.0b013e31824ab7c6

28. Fumery M, Patel NS, Boland BS, Dulai PS, Singh S, Sandborn WJ. Efficacy and Safety of Endoscopic Balloon Dilatation of Ileoanal Pouch Strictures. Inflamm Bowel Dis. 2018;24(6):1316-1320. https://doi.org/10.1093/ibd/ izy006

29. Thompson-Fawcett MW, Marcus V, Redston M, Cohen Z, McLeod RS. Risk of dysplasia in long-term ileal pouches and pouches with chronic pouchitis. Gastroenterology. 2001;121(2):275-81. https://doi.org/10.1053/ gast.2001.26442

30. Derikx LA, Kievit W, Drenth JP, de Jong DJ, Ponsioen CY, Oldenburg B, van der Meulen-de Jong AE, Dijkstra G, Grubben MJ, van Laarhoven CJ, Nagtegaal ID, Hoentjen F; Dutch Initiative on Crohn and Colitis. Prior colorectal neoplasia is associated with increased risk of ileoanal pouch neoplasia in patients with inflammatory bowel disease. Gastroenterology. 2014;146(1):119-28.e1. https://doi. org/10.1053/j.gastro.2013.09.047

31. Pardi DS, D’Haens G, Shen B, Campbell S, Gionchetti P. Clinical guidelines for the management of pouchitis. Inflamm Bowel Dis. 2009;15(9):1424-31. https://doi. org/10.1002/ibd.21039

32. Segal JP, Ding NS, Worley G, Mclaughlin S, Preston S, Faiz OD, Clark SK, Hart AL. Systematic review with metaanalysis: the management of chronic refractory pouchitis with an evidence-based treatment algorithm. Aliment Pharmacol Ther. 2017;45(5):581-592. https://doi. org/10.1111/apt.13905

33. Segal JP, Poo SX, McLaughlin SD, Faiz OD, Clark SK, Hart AL. Long-term follow-up of the use of maintenance antibiotic therapy for chronic antibiotic-dependent pouchitis. Frontline Gastroenterol. 2018;9(2):154-158. https://doi. org/10.1136/flgastro-2017-100913

34. Barreiro-de Acosta M, Marín-Jimenez I, Rodríguez-Lago I, Guarner F, Espín E, Ferrer Bradley I, Gutiérrez A, Beltrán B, Chaparro M, Gisbert JP, Nos P; en representación de GETECCU. Recommendations of the Spanish Working Group on Crohn's Disease and Ulcerative Colitis (GETECCU) on pouchitis in ulcerative colitis. Part 2: Treatment. Gastroenterol Hepatol. 2020;43(10):649-658. https://doi.org/10.1016/j.gastrohep.2020.04.004

35. Gionchetti P, Rizzello F, Helwig U, Venturi A, Lammers KM, Brigidi P, Vitali B, Poggioli G, Miglioli M, Campieri M. Prophylaxis of pouchitis onset with probiotic therapy: a double-blind, placebo-controlled trial. Gastroenterology. 2003;124(5):1202-9. https://doi.org/10.1016/s00165085(03)00171-9
36. Gionchetti P, Rizzello F, Venturi A, Brigidi P, Matteuzzi D, Bazzocchi G, Poggioli G, Miglioli M, Campieri M. Oral bacteriotherapy as maintenance treatment in patients with chronic pouchitis: a double-blind, placebo-controlled trial. Gastroenterology. 2000;119(2):305-9. https://doi. org/10.1053/gast.2000.9370

37. Gosselink MP, Schouten WR, van Lieshout LM, Hop WC, Laman JD, Ruseler-van Embden JG. Delay of the first onset of pouchitis by oral intake of the probiotic strain Lactobacillus rhamnosus GG. Dis Colon Rectum. 2004;47(6):876-84. https://doi.org/10.1007/s10350004-0525-z

38. Tomasz B, Zoran S, Jarosław W, Ryszard M, Marcin G, Robert B, Piotr K, Lukasz K, Jacek P, Piotr G, Przemysław $\mathrm{P}$, Michał D. Long-term use of probiotics Lactobacillus and Bifidobacterium has a prophylactic effect on the occurrence and severity of pouchitis: a randomized prospective study. Biomed Res Int. 2014;2014:208064. https://doi. org/10.1155/2014/208064

39. Pronio A, Montesani C, Butteroni C, Vecchione S, Mumolo G, Vestri A, Vitolo D, Boirivant M. Probiotic administration in patients with ileal pouch-anal anastomosis for ulcerative colitis is associated with expansion of mucosal regulatory cells. Inflamm Bowel Dis. 2008;14(5):662-8. https://doi. org/10.1002/ibd.20369

40. Yasueda A, Mizushima T, Nezu R, Sumi R, Tanaka M, Nishimura J, Kai Y, Hirota M, Osawa H, Nakajima K, Mori $\mathrm{M}$, Ito T. The effect of Clostridium butyricum MIYAIRI on the prevention of pouchitis and alteration of the microbiota profile in patients with ulcerative colitis. Surg Today. 2016;46(8):939-49. https://doi.org/10.1007/s00595015-1261-9

41. Huguet M, Pereira B, Goutte M, Goutorbe F, Dubois A, Bommelaer G, Buisson A. Systematic Review With MetaAnalysis: Anti-TNF Therapy in Refractory Pouchitis and Crohn's Disease-Like Complications of the Pouch After Ileal Pouch-Anal Anastomosis Following Colectomy for Ulcerative Colitis. Inflamm Bowel Dis. 2018;24(2):261268. https://doi.org/10.1093/ibd/izx049

42. Bär F, Kühbacher T, Dietrich NA, Krause T, Stallmach A, Teich N, Schreiber S, Walldorf J, Schmelz R, Büning C, Fellermann K, Büning J, Helwig U; German IBD Study Group. Vedolizumab in the treatment of chronic, antibiotic-dependent or refractory pouchitis. Aliment Pharmacol Ther. 2018;47(5):581-587. https://doi.org/10.1111/ apt.14479

43. Orfanoudaki E, Foteinogiannopoulou K, Koutroubakis IE. Use of vedolizumab in a patient with chronic and refractory pouchitis. Ann Gastroenterol. 2018;31(3):379. https:// doi.org/10.20524/aog.2018.0243

44. Okano S, Yoshimura N, Sako M, Takazoe M. A case of refractory chronic pouchitis successfully treated with tofacitinib. Clin J Gastroenterol. 2020;13(4):560-563. https:// doi.org/10.1007/s12328-020-01108-5

45. Helavirta I, Hyöty M, Huhtala H, Collin P, Aitola P. Longterm functional outcome after restorative proctocolectomy: 
a cross-sectional study. Scand J Gastroenterol. 2018;53(1011):1245-1249. https://doi.org/10.1080/00365521.2018 .1518479

46. Shen B, Yu C, Lian L, Remzi FH, Kiran RP, Fazio VW, Kattan MW. Prediction of late-onset pouch failure in patients with restorative proctocolectomy with a nomogram. J Crohns Colitis. 2012;6(2):198-206. https://doi. org/10.1016/j.crohns.2011.08.006
47. Cold F, Kousgaard SJ, Halkjaer SI, Petersen AM, Nielsen HL, Thorlacius-Ussing O, Hansen LH. Fecal Microbiota Transplantation in the Treatment of Chronic Pouchitis: A Systematic Review. Microorganisms. 2020;8(9):1433. https://doi.org/10.3390/microorganisms8091433 